\title{
Ga Ion-Enhanced and Particle Shape-Dependent Generation of Reactive Oxygen Species in X-ray-Irradiated Composites
}

\author{
W. T. Adams, IV, Michael W. Nolan, ${ }^{\dagger}$ and Albena Ivanisevic*,† \\ ${ }^{\dagger}$ Department of Materials Science and Engineering, North Carolina State University, 911 Partners Way, Raleigh, North Carolina \\ 27695, United States \\ ${ }^{\ddagger}$ Department of Clinical Sciences (College of Veterinary Medicine), and Comparative Medicine Institute, North Carolina State \\ University, 1060 William Moore Drive, Raleigh, North Carolina 27606, United States
}

\section{Supporting Information}

\begin{abstract}
The reported results test the effects of the collective behavior hypothesized to contribute to the production of more reactive oxygen species (ROS) in vitro and result in an enhanced radiosensitization. The role of particle shape in composites with gallium oxyhydroxide $(\mathrm{GaOOH})$ particles and Matrigel is studied. Particles of two different shapes are embedded into the gel to understand only the materials effect on the generation of ROS rather than cell penetrating variations. The paper reports materials characterization by scanning electron microscopy and X-ray diffraction. The stability of the particles within the composite is assessed by quantification of leached metal using inductively coupled plasma mass spectrometry. The amount of ROS in each construct under variable radiation conditions is quantified in the presence and absence of PC12 cells seeded on top of the composites. The viability of cells is also recorded under different in vitro conditions. The collective materials characterization and the results from the bioassays are used to explain the role of anisotropy on the radiosensitization of nanostructures containing Ga. The presence of Ga ions in composites can have a radiosensitizing effect, and the amount of the available $\mathrm{Ga}^{3+}$ determines the magnitude of the radiosensitization. The shape of the particles determines the stability in aqueous solutions and release of $\mathrm{Ga}^{3+}$ that triggers ROS production. The concentration and shape of Ga-containing materials can be combined to generate an additive effect by increasing the amount of available free metal ions in solution. The studies with $\mathrm{GaOOH}$ containing composites enable one to explore the role of key parameters that lead to an increased efficiency of radiation treatments.
\end{abstract}

\section{INTRODUCTION}

The imbalance of reactive oxygen species (ROS) in living systems has been connected to potentially detrimental oxidation of biomolecules that can lead to accelerated cell death. ${ }^{1}$ However, if properly managed the generation of ROS can be incorporated into environmental remediation or therapeutic strategies. Thus, materials and conditions that govern the production of ROS are of interest to a number of research communities addressing biomedical or sustainability challenges. Published work with different nanostructured materials has established that many of their specific characteristics can be linked to variable productions of ROS. ${ }^{2}$ Size and composition of these types of materials have been initially explored. Size of the nanostructures can lead to variable cell uptake and in turn to different productions of ROS. ${ }^{3}$ Shape has also emerged as an additional parameter to consider in explaining variations in ROS levels. ${ }^{4,5}$ For instance, in the case of gold, hexagonal particles produce more ROS compared to spherical and triangular ones during in vitro experiments with epithelial cells, but dose, mass, and volume also greatly impacted particle cell internalization. ${ }^{6}$ In vitro work with carbon materials demonstrated lower ROS levels when spherical shapes were utilized and directly compared to elongated shapes. ${ }^{7}$ In contrast, nanoceria $\left(\mathrm{CeO}_{2}\right)$ evaluated in the presence of macrophages produced no ROS regardless of the shape of the material used. ${ }^{8}$ However, other oxide materials, such as iron oxide, do produce variable ROS levels when rodshaped versus sphere-shaped ones are placed in the presence of macrophages. ${ }^{9}$ Rod versus spherical nanosized $\mathrm{ZnO}$ have also been studied in cancer cell lines and have generated variable ROS species. ${ }^{10}$ The results have been rationalized based on the different stabilities and release of metal ions from each shape.

The production of ROS can be enhanced not only by the presence of specific materials but also by simultaneous exposure to certain environmental conditions, such as radiation. In such situations, the maximum effect can be achieved through the use of a scintillator material. A scintillator is any material that can glow when it is exposed to high-energy particles or photons. ${ }^{11}$ $\mathrm{X}$-rays are used to stimulate a scintillator to emit light. The scintillator material is sometimes described as an X-ray

Received: March 19, 2018

Accepted: May 3, 2018

Published: May 15, 2018 
Scheme 1. Representation of the Approach Used to Test the Role of Shape of GaOOH Particles on PC12 Cell Viability and ROS Production

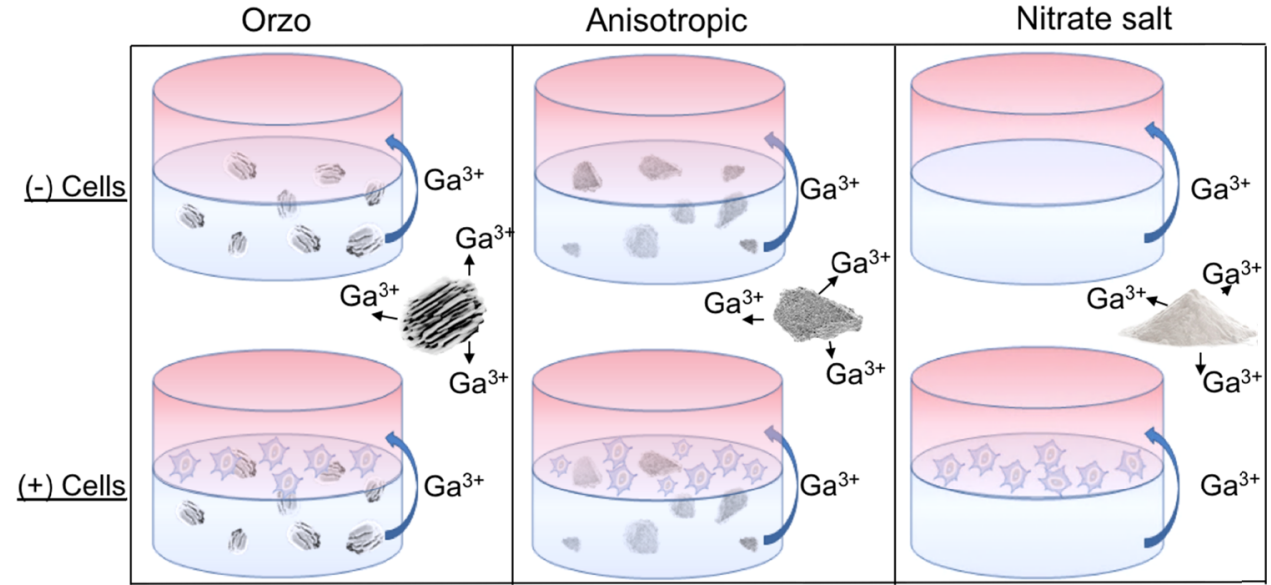

transducer that facilitates the generation of ${ }^{1} \mathrm{O}_{2}$ through an energy-transfer process. A number of medical imaging techniques rely on scintillators, and advancements in recent years have been largely due to materials discovery as well as instrumentation improvements. ${ }^{12}$ From a materials prospective, the utility of scintillators is based on the fact that they enable the conversion of X-rays to $\mathrm{UV} /$ visible photons within 1 ps. The structure, size, and composition of scintillators can enhance the desired strong luminescence emission. ${ }^{13}$ Radiation sensitization or radiosensitization refers to a collection of strategies to enhance the effect of radiation exposure. ${ }^{14}$ With respect to biomaterials design that entails the generation of more secondary electrons and increased production of ROS that lead to improved radiation efficacy. ${ }^{15}$ Quantification of the production of ROS after X-ray exposure is one way to benchmark radiosensitization. ${ }^{16}$ Many material systems have been studied to assess their ability to act as radiosensitizers. Promising results with a number of high Z-materials have been published, and their references are too numerous to list. However, studies traditionally do not compare architectural configurations with different sizes, shapes, and compositions. Designing a biomaterials system that enables such comparisons will facilitate the identification of the most suitable candidates for enhanced radiosensitization that can be further tested in clinically relevant models.

In this paper, we examine the role of shape in composites with gallium oxyhydroxide $(\mathrm{GaOOH})$ particles and Matrigel. The current work builds on prior promising results that demonstrated that the presence of a scintillator material inside a scaffold triggered different cellular responses when radiation was used. ${ }^{17,18}$ These studies performed by Berg et al. have demonstrated the behavior of $\mathrm{GaOOH}$ nanoparticles in cell free environments of both water and cell growth media. We now study the effect of $\mathrm{GaOOH}$ particle anisotropy in a composite. The particles are embedded into the gel to understand only the materials effect on the generation of ROS rather than cell penetrating variations, Scheme 1. Gallium containing nanostructures were chosen for their favorable scintillating properties, documented therapeutic potential of $\mathrm{Ga}^{19}$ and recent renewed interest to improve cellular uptake and anticancer activity of Ga-coordinating complexes. ${ }^{20}$ The inspiration for the design of this current study is based on key prior observations. ${ }^{17,18}$ We observed that in Ga-containing composites the ROS production is chemical stemming due to the presence of the GaOOH. Therefore, we wanted to test if different shapes of particles with variable stabilities will produce different ROS amounts. In addition, the design depicted in Scheme 1 is intended to test if the presence of the any amount of $\mathrm{GaOOH}$ can enhance the production of ROS species after variable radiation conditions. We note that in prior work we have also assessed the Ga toxicity using an extremely watersoluble salt: $\mathrm{Ga}\left(\mathrm{NO}_{3}\right)_{3}{ }^{18}$ Previously, we recorded no concentration dependence on toxicity. In this work, we test the effects of the collective behavior hypothesized to contribute to the production of more ROS and result in an enhanced radiosensitization. The $\mathrm{GaOOH}$ particles are characterized by scanning electron microscopy (SEM) and X-ray diffraction (XRD). We evaluate the stability of $\mathrm{GaOOH}$ in solution by recording the amount of metal leached using inductively coupled plasma mass spectrometry (ICP-MS). The amount of ROS in each construct under variable radiation conditions is quantified in the presence and absence of cells seeded on top of the composites. The viability of cells is also recorded under different in vitro conditions. The collective materials characterization and the results from the bioassays are used to explain the role of anisotropy on the radiosensitization of nanostructures containing $\mathrm{Ga}$.

\section{RESULTS AND DISCUSSION}

We focus on understanding of how to enhance the radiosensitization effect during the fabrication of the composite rather than exploring ideas to increase the effect via better uptake of particles into cells. We prepared three composites with different arrangements, shown in Scheme 1, to test the following hypotheses: (1) composition and amount of Zmaterial in the composite determine the magnitude of the radiosensitization; (2) anisotropy of the nanostructured material in the composite has an effect on the generation of ROS; (3) material composition and anisotropy can be used to generate additive or cooperative effects. We synthesized two different shapes of $\mathrm{GaOOH}$ and also used commercially available gallium salt, $\mathrm{Ga}\left(\mathrm{NO}_{3}\right)_{3}$ that is highly soluble in water. We chose these Ga-containing materials because even small quantities of Ga can have noticeable impact. It is known that gallium ions can bind to transferrin receptors and be taken specifically and nonspecifically into cells. ${ }^{19}$ This can result in severe disturbance of iron homeostasis and have adverse effects. $^{21}$ The amount and shape of the Ga-based material are 


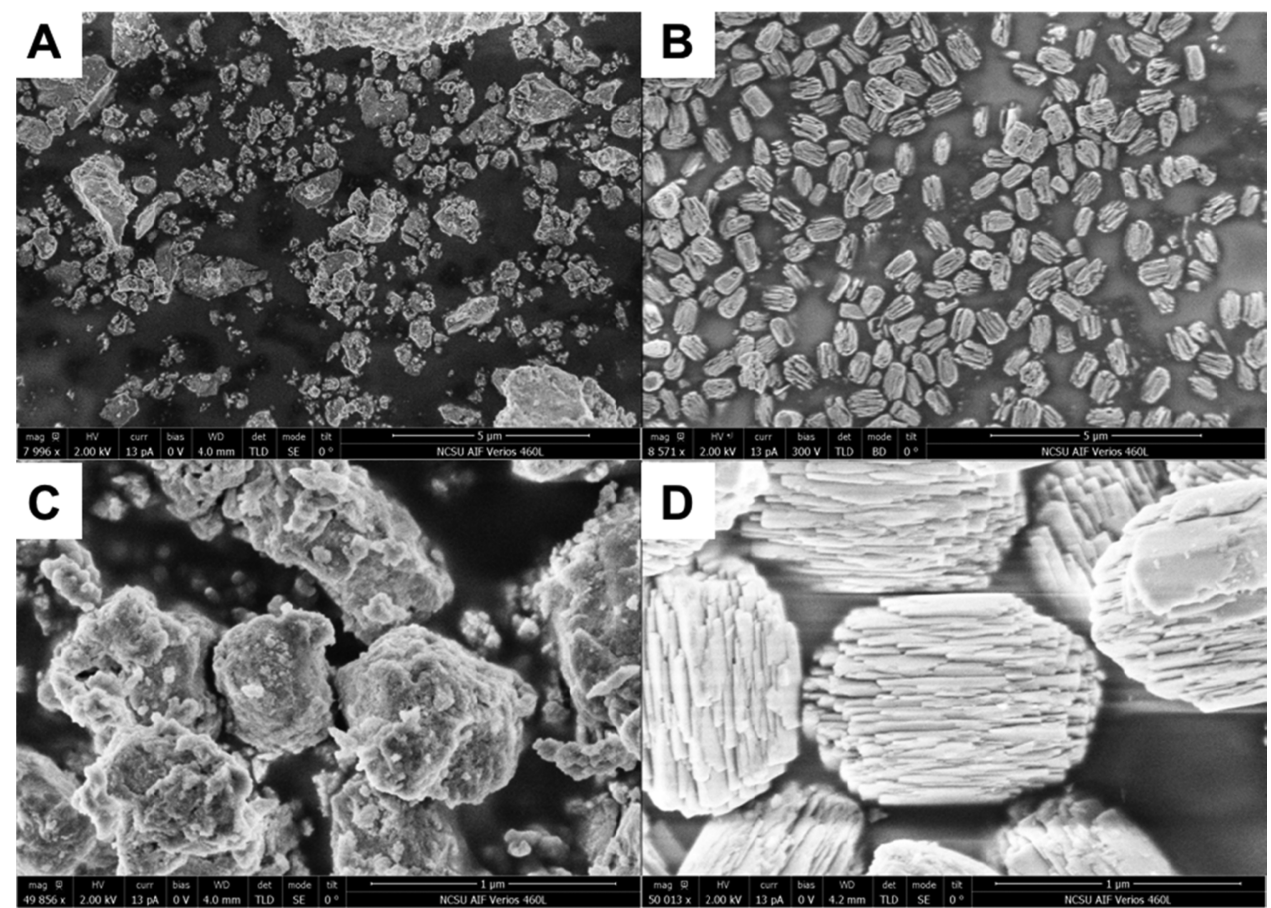

Figure 1. Representative SEM images of the different shapes of GaOOH used in the study: (A) anisotropic and (B) orzo. (C) and (D) show zoomed in structures for the anisotropic and orzo shapes, respectively.
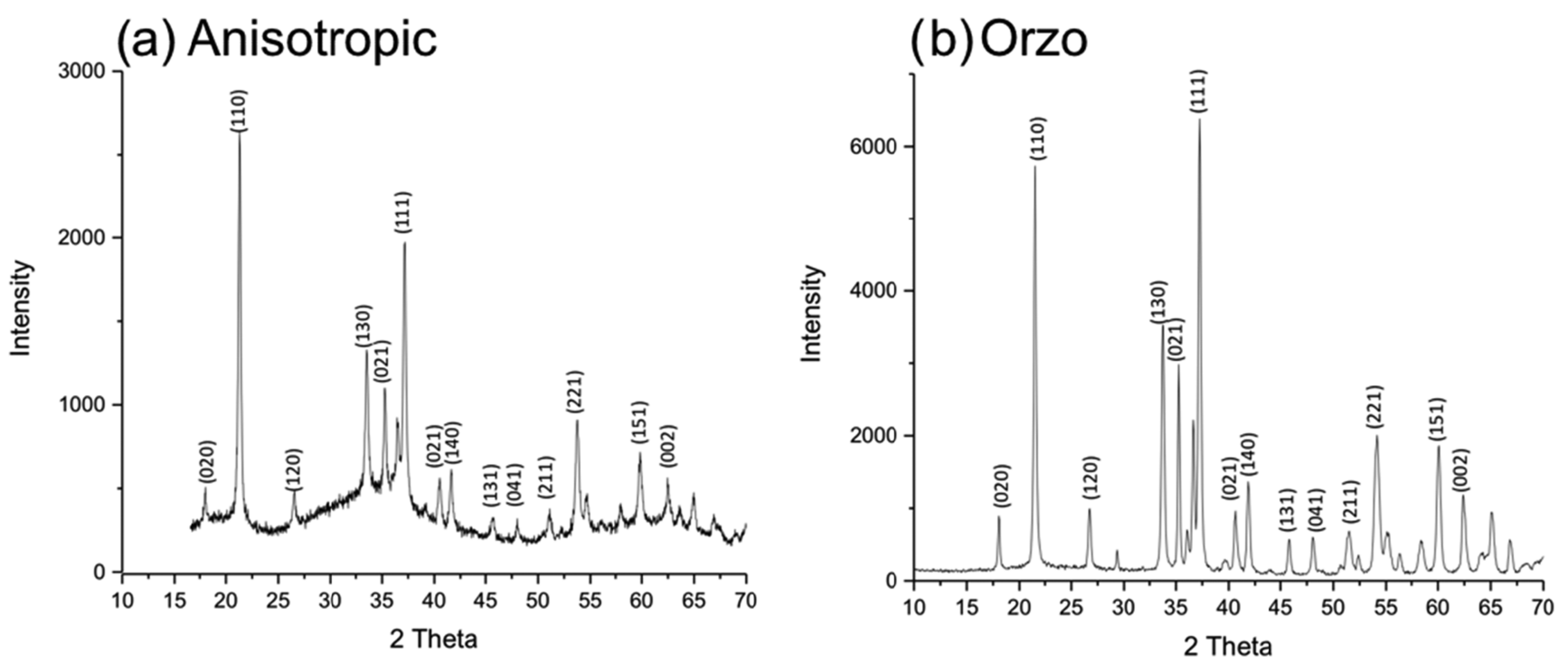

Figure 2. XRD characterization of the different Ga-containing materials used in the study: (a) anisotropic GaOOH particles and (b) orzo GaOOH particles.

controlled during the composite manufacturing. The composite fabrication requires no specialized equipment and only easy processing that relies on different Ga mass quantities to vary the properties of the composite. PC12 cells were used to study the production of ROS before and after radiation. During all testing, the cells were seeded on top of the $\mathrm{GaOOH} /$ Matrigel composites to avoid endocytosis of $\mathrm{GaOOH}$-nanostructured material. No visible particles were identified on the top of the interface. In the experimental setup, we also performed experiments in the absence of cells. This setup permitted us to understand the ROS changes induced by the Ga-based materials in the composite after radiation rather than changes as a result of transformations due to dynamic biological processes driven by the cells.

A number of research groups have published reports on how to synthesize $\mathrm{GaOOH}$ via solvothermal procedures. ${ }^{22-24} \mathrm{We}$ choose to utilize the microwave synthesis method because: the synthesis can be finished in less than an hour; the starting material, $\mathrm{GaNO}_{3}$, is commercially available and affordable for a large-scale synthesis; the size and shape of the nanostructured product can be varied by tuning the reaction conditions; and the resulting particles do not require any stabilizing ligands. In a typical synthesis run, we dissolved $\mathrm{GaNO}_{3}$ in water to create a $0.1 \mathrm{M}$ solution. We increased the $\mathrm{pH}$ to 8 by slowing the addition of $\mathrm{NaOH}$. That solution is then loaded into a glass 

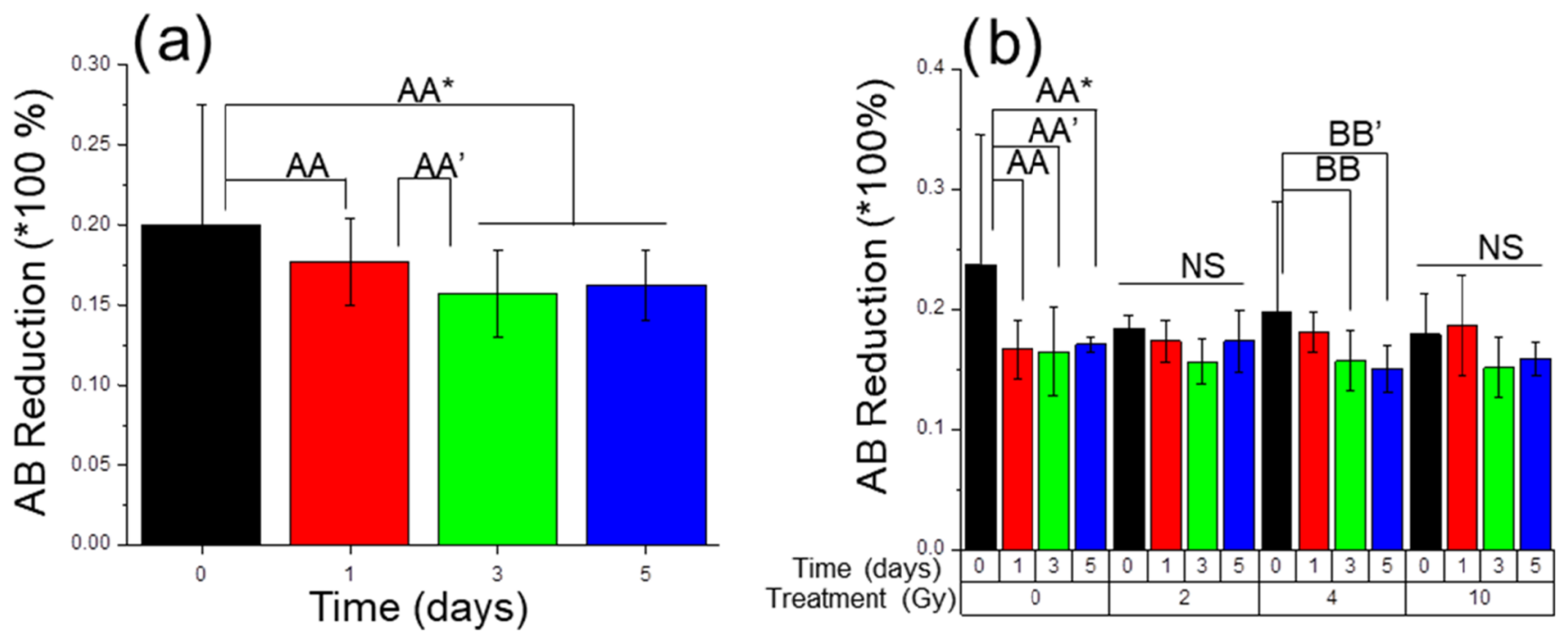

Figure 3. Cell viability assay results in the absence of $\mathrm{GaOOH}$ material: (a) $\mathrm{AB}$ assay conducted in the presence of no material, just Matrigel; (b) $\mathrm{AB}$ assay conducted in the presence of no material and after the indicated radiation treatment.

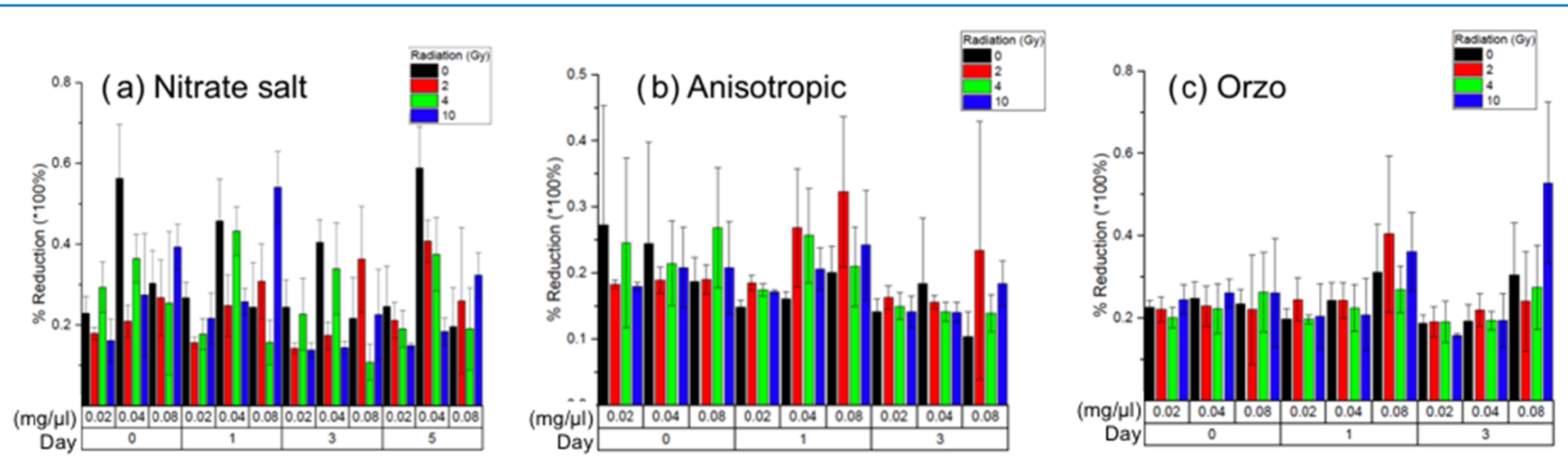

Figure 4. Summary of the cell viability assays performed with Alamar Blue after the indicated times in days, exposure to different amounts of radiation and in the presence of the specified concentrations of (a) nitrate salt; (b) anisotropic $\mathrm{GaOOH}$; and (c) orzo $\mathrm{GaOOH}$.

vessel and placed into a microwave synthesis system and heated to $120{ }^{\circ} \mathrm{C}$ for $20 \mathrm{~min}$. The product that forms is fluffy and white in color. This product is washed with water and vacuumdried with no heat. During the entire procedure, no organic ligands are used to control the size and shape of the particles. One can change the shape by simply omitting the use of heat to allow the formation of $\mathrm{GaOOH}$. The quality of the particles was assessed with a number of characterization techniques. Thorough examination of particle shape and morphology was performed with SEM analysis and representative images are shown in Figure 1. The SEM characterization was performed to assess size and shape and not to assess aggregation behavior. We termed one shape of particles as orzo due to its specific elongated geometry with the appearance of stacked (lenticular) plates that comprise the overall ovoid shape. The other shape is referred to as anisotropic and was observed to be granular with random nanoscale features on each side of its blocky, subangular structure. XRD was used to track the transformation of $\mathrm{GaNO}_{3}$ (an amorphous material) to crystalline $\mathrm{GaOOH}$ by looking at the shape (broad for amorphous and sharp for crystalline) of the peaks in the spectrum, Figure 2. Use of Scherrer's equation provides a way to compare the two types of particles synthesized. Because peak width is inversely proportional to crystalline size, we analyzed the data collected for both shapes and the peak at the (110) direction. Calculations done using Scherrer's equation and the peak at the (110) direction showed that the crystalline grains for the orzo shape are $\sim 49$ $\mathrm{nm}$ and for the anisotropic one to be $\sim 53 \mathrm{~nm}$. In addition, we confirmed that the diffraction patterns on Figure 1 can be assigned to the orthorhombic $\mathrm{GaOOH}$ structure and are in agreement with literature reports. $^{25}$

Nanostructured-Matrigel composites for all testing of the stated hypothesis were prepared by mixing either the $\mathrm{GaOOH}$ particles or the $\mathrm{Ga}\left(\mathrm{NO}_{3}\right)_{3}$ salt with Matrigel and cell medium. All of the compositions were placed in standard 96-well plates. The compositions contained the following concentrations of Ga-based material: 0.02, 0.04, and $0.08 \mathrm{mg} / \mu \mathrm{L}$ (See Supporting Information for plate design for each bioassay). Each plate was irradiated using a $6 \mathrm{MV}$ photon beam from a clinical linear accelerator (Novalis TX; Varian Medical Systems, Palo Alto, CA). Prior to the irradiation experiments, the plate was surrounded by at least $1 \mathrm{~cm}$ of solid, homogeneous, tissueequivalent bolus material (density of $1.03 \mathrm{~g} / \mathrm{cm}^{3}$ ). The setup is essential to achieve buildup of lateral electronic equilibrium. Prior to every experiment, each plate was placed at least $2 \mathrm{~cm}$ from the edge of the radiation field to minimize penumbra effects. In addition, we covered the cell culture dishes with 8.9 $\mathrm{cm}$ solid water phantom material, which was necessary to guarantee the maximal field flatness. The output rate was set to $200 \mathrm{MU} \mathrm{min}^{-1}\left(\mathrm{SAD}=100 \mathrm{~cm}, d=10 \mathrm{~cm}, A=15 \times 15 \mathrm{~cm}^{2}\right)$. 

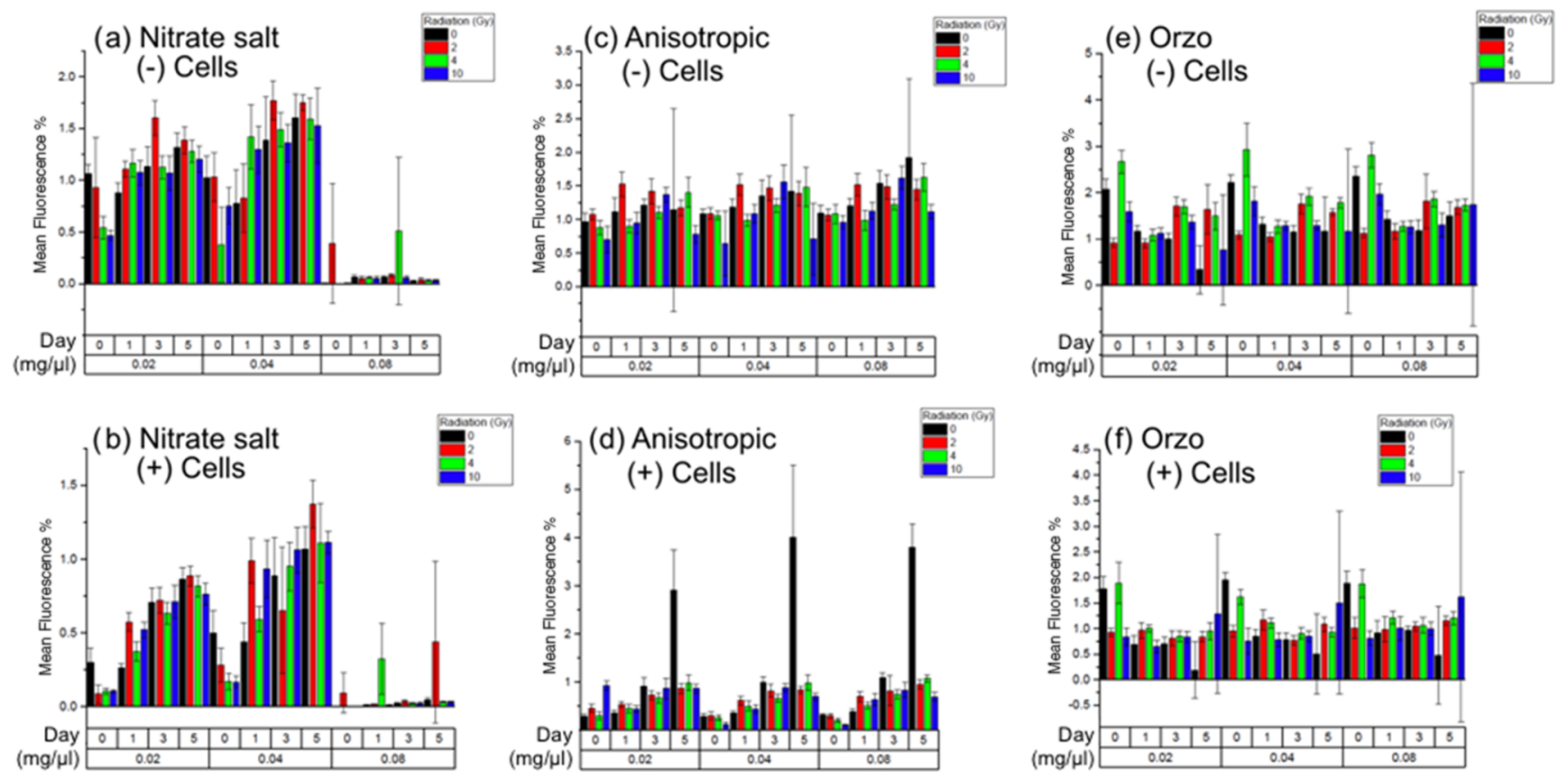

Figure 5. Summary of the ROS assays performed with DCFH-DA after the indicated times in days, exposure to different amounts of radiation and in the presence of the specified concentrations of (a) nitrate salt; (b) nitrate salt and PC12 cells seeded on top of the Ga-containing composite; (c) anisotropic $\mathrm{GaOOH}$; (d) anisotropic $\mathrm{GaOOH}$ and PC12 cells seeded on top of the Ga-containing composite; (e) orzo $\mathrm{GaOOH}$; and (f) orzo $\mathrm{GaOOH}$ and PC12 cells seeded on top of the Ga-containing composite.

The samples inside the cell culture dishes received total doses of 2, 4, or $10 \mathrm{~Gy}$ in a single fraction.

We chose to utilize the PC12 cell line due to the ease of handling and the abundance of studies published in the literature utilizing the same in vitro protocols. ${ }^{26}$ The procedure for the growth of PC12 cells utilized Dulbecco's modified Eagle medium (DMEM) high glucose formulation with $12.5 \%$ horse serum, $2.5 \%$ fetal bovine serum, and $1 \%$ penicillinstreptomycin. The conditions for the incubation were $37{ }^{\circ} \mathrm{C}$ and $5 \% \mathrm{CO}_{2}$ in a humidified incubator and were maintained throughout all experiments. The parameters for each trial were $7.5 \times 10^{4} \mathrm{cell} / \mathrm{mL}$ density and $0.5 \mathrm{~mL}$ per $100 \mathrm{~mm}$ plate. We allowed the PC12 cells to adhere for $18 \mathrm{~h}$ prior to beginning each trial. Control experiments were performed on gels with no Ga material. We assessed cell survival (Alamar Blue assay) and ROS generation $\left(2^{\prime}, 7^{\prime}\right.$-dichlorofluorescin diacetate (DCFHDA) assay). Figures 3 and 4 summarize the results from the cell assays. Due to the number of parameters and comparisons, all statistical analyses are reported in the Supporting Information. Here, we present a summary of the statistically significant interactions that were observed along with key conclusions we made based on the recorded results and subsequent statistical assessment.

In experiments performed on Matrigel with no Gacontaining materials, we observed a small decrease in cell viability with time when the PC12 cells were not exposed to any radiation treatments. Some cell death is expected as the amount of cells grows with the progress of duration of the experiment, as seen in Figure 3a. When these samples were exposed to radiation, increasing radiation exposure resulted in no significant changes in cell survival as the days of the experiments progressed, Figure $3 \mathrm{~b}$. We observed very different results in viability when the three types of Ga-containing materials were added to the composite, Figure 4. The Ga-salt yielded statistically significant decrease in viability as both the concentration and the radiation dose increased. In the case of the orzo particles, the viability of PC12 cells changed only at higher concentrations, and the amount of radiation did not influence the cell survival significantly. In contrast, the anisotropic particles contributed to a decrease in cell viability based on increasing concentration and a smaller decrease based on higher doses of radiation treatment. Literature reports have established that the levels of tolerance for $\mathrm{Ga}$ ions in different living systems are yet to be established, but evidence points to the conclusion that the lethal toxicity of $\mathrm{Ga}^{3+}$ is significantly lower than other metal ions. ${ }^{27}$ Our viability results support this conclusion because we observed significant changes only at increased concentrations. The radiation exposure decreased the viability of the PC12 when a significant amount of Ga was present in the system supporting the notion that this metal can have a radiosensitizing effect.

We quantified the amount of ROS under different radiation conditions in the presence and absence of cells, Figure 5. The presence of ROS causes breaks in the DNA structure and leads to cell apoptosis and is used as a measure of radiosensitization. The fluorescence at different time points (days) was recorded. The DCFH-DA was dissolved in DMEM at a concentration of $60 \mu \mathrm{M}$. For each measurement, the cell media was aspirated off and $1 \mathrm{~mL}$ of the DCFH-DA solution was added. An incubation time of $30 \mathrm{~min}$ was used prior to the fluorescence measurements with a Tecan GENios microplate reader. The excitation filter and emission filters were at 485 and $535 \mathrm{~nm}$, respectively. The use of DCFH-DA in cell free experiments has been the subject of other published work that showed that with proper control experiments one can utilize the results obtained by this assay to understand factors that influence the generation of ROS in solution. ${ }^{28,29}$ We first consider the data obtained in the absence of cells, first row of plots on Figure 5. In the case of the gallium salt, we recorded an increase in ROS production with an increase in concentration and eventually saw a saturation 
effect at the highest concentration. When the samples containing the gallium salt were exposed to radiation at different doses, we only observed an initial significant change in ROS production, and no change was observed as the days of the experiment progressed. In the case of the orzo particles, we observed a decrease in ROS production with higher concentrations and higher doses in radiation. However, with the anisotropic particles higher concentrations and higher doses in radiation yielded more production of ROS. Overall, the results we recorded in the absence of cells support the notion that the orzo particles are more stable in solution and release less gallium ions that can cause the production of ROS. The data recorded with the anisotropic shape gave trends similar to the ones collected with the water-soluble $\mathrm{Ga}\left(\mathrm{NO}_{3}\right)_{3}$ supporting a conclusion that this shape of $\mathrm{GaOOH}$ is highly unstable in aqueous solutions, which can induce ROS production.

The ROS assay was performed in the same manner after cells were seeded on the same composites and exposed to the same radiation conditions, second row of plots on Figure 5. We observed the same overall comparative trends in ROS production, as we did when there were no cells present, though the amount of ROS measured was different compared to the data when no cells were present. The results from both bioassays suggest that the availability of $\mathrm{Ga}$ ions in solution is the main reason for the observed changes after radiation exposure with respect to cell viability as well as ROS production. We tested the stability of the two types of $\mathrm{GaOOH}$ by incubating them in cell media, filtering the particles out, and measuring the amount of Ga leached by ICP-MS. All of the data along with standard deviations are plotted and presented in the Supporting Information. Table 1 shows a

Table 1. Summary of Differences in the Amount of Ga Leached Compared to the Initial Value on Day 0 Immediately after Placing the Particles in Solution

$\begin{array}{lcc}\text { GaOOH type } & \text { day } 1(\%) & \text { day } 3(\%) \\ \text { orzo } & -24 \pm 0.5 & -52 \pm 2.2 \\ \text { anisotropic } & +149 \pm 26.2 & +454 \pm 14.8\end{array}$

comparison between the two trends of behavior we recorded. We observed that the anisotropic particles continued to release $\mathrm{Ga}$ in solution, as the days of the experiment progressed. In contrast, the amount of Ga released from the orzo particles drastically decreases, as the material was incubated in solution for 3 days. The data in Table 1 support the notion that placement of the orzo particles in solution for longer periods of time leads to passivation of their surface. Taken in sum the data collected by ICP-MS supports the conclusions from the ROS and viability assays and confirm that two types of particles have different stabilities in solution. Thus, the overall trends in ROS production based on concentration and radiation conditions were governed by particle stability. Leached amount of Ga coupled with radiation dose can be used to generate different amounts of ROS during in vitro experiments.

\section{CONCLUSIONS}

In summary, we demonstrate that the presence of $\mathrm{Ga}$ ions in composites can have a radiosensitizing effect during in vitro experiments with PC12 cells. We confirmed our hypothesis that the amount of the available $\mathrm{Ga}^{3+}$ determines the magnitude of the radiosensitization. With respect to shape of the $\mathrm{GaOOH}$ particles, one observes changes in the generation of ROS, which are related to the stability of the specific shape in aqueous solutions. Further, our results support the notion that concentration and shape of Ga-containing materials can be combined to generate an additive effect by increasing the amount of available free metal ions in solution. The metal ions contribute to the greatest generation of ROS after exposure to ionizing radiation. The studies with $\mathrm{GaOOH}$ containing composites enable one to explore the role of key parameters that lead to an increased efficiency of radiation treatments. The reported results are currently being considered in our laboratories for further translational biomaterials approaches.

\section{EXPERIMENTAL SECTION}

4.1. Materials. The gallium nitrate hydrate (289892) was obtained from Sigma-Aldrich. Phenol red free Matrigel (354262) and sodium hydroxide (UN1823) were obtained from Fisher Scientific. The Alamar Blue assay (DAL 1025) was supplied by ThermoFisher Scientific. The ROS assay uses $2^{\prime}, 7^{\prime}$ dichlorofluorescin diacetate (DCFH-DA) and was utilized as received from Sigma-Aldrich.

4.2. Ga Particle Fabrication. The orzo GaOOH nanoparticles were synthesized via a microwave reaction based on an experimental procedure reported by Pearce et al. ${ }^{30}$ The anisotropic $\mathrm{GaOOH}$ nanoparticles were synthesized by precipitating $\mathrm{GaOOH}$ from solution with no additional microwave or thermal treatment.

4.3. Composite Preparation. The Matrigel composite was prepared by following the thin-gel protocol provided by Corning and diluting to a protein concentration of $3 \mathrm{mg} / \mathrm{mL}$. The gallium bearing material was mixed with Matrigel just after pipetting into cell culture plates to ensure homogeneity before gelation.

4.4. SEM Characterization. All samples were evaluated using the FEI Verios 460L in AIF. The Everhart-Thornley detector was used in backscatter mode with an accelerating voltage of $2 \mathrm{kV}$ and a sample current of $13 \mathrm{pA}$.

4.5. XRD Characterization. XRD analysis was performed with a Rigaku SmartLab X-ray diffractometer. Powder diffraction was done with a $\mathrm{Cu} \mathrm{K} \alpha \mathrm{X}$-ray source at $40 \mathrm{kV}$ and $40 \mathrm{~mA}$ with Bragg-Brentano optical setup.

4.6. Cell Culture and Bioassays. Culturing of PC12 cells was done using a sterile technique and a protocol described in prior work. ${ }^{31}$ Cells were kept in an incubator at $37^{\circ} \mathrm{C}$, and the cell media was replenished every $48 \mathrm{~h}$. The bioassays were carried out using the manufacturer's protocol, and details for each one have been reported elsewhere. ${ }^{32}$

4.7. ICP-MS. The samples incubated in the two shapes of $\mathrm{GaOOH}$ was filtered and analyzed for gallium amount with a Perkin Elmer Sciex Elan DRCII ICP-MS. Prior to any data collection, a calibration was done with gallium 69. The solutions were also diluted before the analysis, and the dilutions are factored in the final values of the leached Ga.

4.8. Radiation Exposure Experiments. All plates were irradiated with $6 \mathrm{MV}$ photon beam from a clinical linear accelerator (Novalis TX; Varian Medical Systems, Palo Alto, CA). Experimental details have been detailed in Berg et al. ${ }^{33}$

4.9. Statistical Analysis. All statistical analyses were conducted using OriginPro 2016 (b9.3.1.273). One-way and two-way analysis of variances were used to find differences between the experimental conditions with a significance level of 0.05 . 


\section{ASSOCIATED CONTENT}

\section{S Supporting Information}

The Supporting Information is available free of charge on the ACS Publications website at DOI: 10.1021/acsomega.8b00524.

Additional experimental details, statistical analysis, and characterization data (PDF)

\section{AUTHOR INFORMATION}

\section{Corresponding Author}

*E-mail: ivanisevic@ncsu.edu.

\section{ORCID ${ }^{\circ}$}

Albena Ivanisevic: 0000-0003-0336-1170

\section{Notes}

The authors declare no competing financial interest.

\section{ACKNOWLEDGMENTS}

This work was performed in part at the Analytical Instrumentation Facility (AIF) at North Carolina State University, which is supported by the State of North Carolina and the National Science Foundation (award number ECCS1542015). The AIF is a member of the North Carolina Research Triangle Nanotechnology Network (RTNN), a site in the National Nanotechnology Coordinated Infrastructure (NNCI). The work was performed in part at the Environmental and Agricultural Testing Service Laboratory (EATS), Department of Crop and Soil Sciences at North Carolina State University.

\section{REFERENCES}

(1) Khaing Oo, M. K.; Yang, Y.; Hu, Y.; Gomez, M.; Du, H.; Wang, H. Gold Nanoparticle-Enhanced and Size-Dependent Generation of Reactive Oxygen Species from Protoporphyrin IX. ACS Nano 2012, 6, 1939-1947.

(2) Wyrwoll, A. J.; Lautenschläger, P.; Bach, A.; Hellack, B.; Dybowska, A.; Kuhlbusch, T. A. J.; Hollert, H.; Schäffer, A.; Maes, H. M. Size Matters-The Phototoxicity of $\mathrm{TiO} 2$ Nanomaterials. Environ. Pollut. 2016, 208, 859-867.

(3) Cardillo, D.; Tehei, M.; Hossain, M. S.; Islam, M. M.; Bogusz, K.; Shi, D.; Mitchell, D.; Lerch, M.; Rosenfeld, A.; Corde, S.; Konstantinov, K. Synthesis-Dependent Surface Defects and Morphology of Hematite Nanoparticles and Their Effect on Cytotoxicity in Vitro. ACS Appl. Mater. Interfaces 2016, 8, 5867-5876.

(4) Schaeublin, N. M.; Braydich-Stolle, L. K.; Maurer, E. I.; Park, K.; MacCuspie, R. I.; Afrooz, A. R. M. N.; Vaia, R. A.; Saleh, N. B.; Hussain, S. M. Does Shape Matter? Bioeffects of Gold Nanomaterials in a Human Skin Cell Model. Langmuir 2012, 28, 3248-3258.

(5) Laha, D.; Pramanik, A.; Laskar, A.; Jana, M.; Pramanik, P.; Karmakar, P. Shape-Dependent Bactericidal Activity of Copper Oxide Nanoparticle Mediated by DNA and Membrane Damage. Mater. Res. Bull. 2014, 59, 185-191.

(6) Tian, F.; Clift, M. J. D.; Casey, A.; del Pino, P.; Pelaz, B.; Conde, J.; Byrne, H. J.; Rothen-Rutishauser, B.; Estrada, G.; de la Fuente, J. M.; Stoeger, T. Investigating the Role of Shape on the Biological Impact of Gold Nanoparticles in Vitro. Nanomedicine 2015, 10, 26432657.

(7) Kang, S.; Kim, J.-E.; Kim, D.; Woo, C. G.; Pikhitsa, P. V.; Cho, M.-H.; Choi, M. Comparison of Cellular Toxicity between MultiWalled Carbon Nanotubes and Onion-like Shell-Shaped Carbon Nanoparticles. J. Nanopart. Res. 2015, 17, No. 378.

(8) Forest, V.; Leclerc, L.; Hochepied, J.-F.; Trouvé, A.; Sarry, G.; Pourchez, J. Impact of Cerium Oxide Nanoparticles Shape on Their in Vitro Cellular Toxicity. Toxicol. In Vitro 2017, 38, 136-141.

(9) Lee, J. H.; Ju, J. E.; Kim, B. I.; Pak, P. J.; Choi, E.-K.; Lee, H.-S.; Chung, N. Rod-Shaped Iron Oxide Nanoparticles Are More Toxic than Sphere-Shaped Nanoparticles to Murine Macrophage Cells. Environ. Toxicol. Chem. 2014, 33, 2759-2766.

(10) Bhattacharya, D.; Bhattacharyya, A.; Karmakar, P. Evaluation of Different Oxidative Stress Parameters and Apoptosis in Human Cervical Cancer Cells Exposed to Rod and Spherical Shaped Zinc Oxide Nanoparticles. Bionanoscience 2016, 6, 1-14.

(11) Kamkaew, A.; Chen, F.; Zhan, Y.; Majewski, R. L.; Cai, W. Scintillating Nanoparticles as Energy Mediators for Enhanced Photodynamic Therapy. ACS Nano 2016, 10, 3918-3935.

(12) Ronda, C.; Wieczorek, H.; Khanin, V.; Rodnyi, P. ReviewScintillators for Medical Imaging: A Tutorial Overview. ECS J. Solid State Sci. Technol. 2016, 5, R3121-R3125.

(13) Chen, H.; Moore, T.; Qi, B.; Colvin, D. C.; Jelen, E. K.; Hitchcock, D. A.; He, J.; Mefford, O. T.; Gore, J. C.; Alexis, F.; Anker, J. N. Monitoring $\mathrm{pH}$-Triggered Drug Release from Radioluminescent Nanocapsules with X-Ray Excited Optical Luminescence. ACS Nano 2013, 7, 1178-1187.

(14) Fan, W.; Bu, W.; Shi, J. On The Latest Three-Stage Development of Nanomedicines Based on Upconversion Nanoparticles. Adv. Mater. 2016, 28, 3987-4011.

(15) Retif, P.; Pinel, S.; Toussaint, M.; Frochot, C.; Chouikrat, R.; Bastogne, T.; Barberi-Heyob, M. Nanoparticles for Radiation Therapy Enhancement: The Key Parameters. Theranostics 2015, 5, 1030-1045.

(16) Bulin, A.-L.; Truillet, C.; Chouikrat, R.; Lux, F.; Frochot, C.; Amans, D.; Ledoux, G.; Tillement, O.; Perriat, P.; Barberi-Heyob, M.; Dujardin, C. X-Ray-Induced Singlet Oxygen Activation with Nanoscintillator-Coupled Porphyrins. J. Phys. Chem. C 2013, 117, 2158321589.

(17) Berg, N. G.; Pearce, B. L.; Rohrbaugh, N.; Jiang, L.; Nolan, M. W.; Ivanisevic, A. Gallium Containing Composites as a Tunable Material to Understand Neuronal Behavior under Variable Stiffness and Radiation Conditions. Mater. Sci. Eng., C 2017, 71, 317-321.

(18) Berg, N. G.; Pearce, B. L.; Snyder, P. J.; Rohrbaugh, N.; Nolan, M. W.; Adhikari, P.; Khan, S. A.; Ivanisevic, A. Interfaces with Tunable Mechanical and Radiosensitizing Properties. ACS Appl. Mater. Interfaces 2016, 8, 21956-21961.

(19) Chitambar, C. R. Gallium and Its Competing Roles with Iron in Biological Systems. Biochim. Biophys. Acta, Mol. Cell Res. 2016, 1863, 2044-2053.

(20) Pribisko, M.; Palmer, J.; Grubbs, R. H.; Gray, H. B.; Termini, J.; Lim, P. Cellular Uptake and Anticancer Activity of Carboxylated Gallium Corroles. Proc. Natl. Acad. Sci. U.S.A. 2016, 113, E2258E2266.

(21) Schreinemachers, D. M.; Ghio, A. J. Article Commentary: Effects of Environmental Pollutants on Cellular Iron Homeostasis and Ultimate Links to Human Disease. Environ. Health Insights 2016, 10, No. EHI-S36225.

(22) Wu, S.; Zhang, J.; Shi, L.; Tang, S.; Li, Y.; Jiang, L.; Cui, Q. Template-Free Synthesis of Alpha-GaOOH Hyperbranched Nanoarchitectures via Crystal Splitting and Their Optical Properties. RSC Adv. 2014, 4, 8209-8215.

(23) Muruganandham, M.; Suri, R.; Wahed, M. S. M. A.; Sillanpaa, M.; Ahmmad, B.; Lee, G.-J.; Wu, J. J. Solvothermal Synthesis of Mesoporous Alpha-GaOOH Semi-Nanospheres. Mater. Lett. 2013, $111,137-139$.

(24) Muruganandham, M.; Amutha, R.; Wahed, M. S. M. A.; Ahmmad, B.; Kuroda, Y.; Suri, R. P. S.; Wu, J. J.; Sillanpää, M. E. T. Controlled Fabrication of $\alpha-\mathrm{GaOOH}$ and $\alpha$-Ga2O3 Self-Assembly and Its Superior Photocatalytic Activity. J. Phys. Chem. C 2012, 116, 4453.

(25) Ganguly, B. N.; Verma, V.; Chatterjee, D.; Satpati, B.; Debnath, S.; Saha, P. Study of Gallium Oxide Nanoparticles Conjugated with $\beta$ Cyclodextrin: An Application To Combat Cancer. ACS Appl. Mater. Interfaces 2016, 8, 17127-17137.

(26) Koh, H. S.; Yong, T.; Chan, C. K.; Ramakrishna, S. Enhancement of Neurite Outgrowth Using Nano-Structured Scaffolds Coupled with Laminin. Biomaterials 2008, 29, 3574-3582.

(27) Betoulle, S.; Etienne, J.-C.; Vernet, G. In vivo and in vitro modulation of carp ( cyprinus carpio 1.) phagocyte oxidative burst 
activity by gallium. J. Toxicol. Environ. Health, Part A 2002, 65, 603615.

(28) Korystov, Y. N.; Shaposhnikova, V. V.; Korystova, A. F.; Emel'yanov, M. O. Detection of Reactive Oxygen Species Induced by Radiation in Cells Using the Dichlorofluorescein Assay. Radiat. Res. 2007, 168, 226-232.

(29) Boulton, S.; Anderson, A.; Swalwell, H.; Henderson, J. R.; Manning, P.; Birch-Machin, M. A. Implications of Using the Fluorescent Probes, Dihydrorhodamine 123 and $2^{\prime}, 7^{\prime}$-Dichlorodihydrofluorescein Diacetate, for the Detection of UVA-Induced Reactive Oxygen Species. Free Radical Res. 2011, 45, 115-122.

(30) Pearce, B. L.; Berg, N. G.; Rahn, M. S.; Ivanisevic, A. In Situ and Ex Situ Functionalization of Nanostructured Gallium Oxy-Hydroxide with a Porphyrin Dye. Scanning 2016, 38, 671-683.

(31) Snyder, P. J.; Kirste, R.; Collazoa, R.; Ivanisevic, A. Nanoscale Topography, Semiconductor Polarity and Surface Functionalization: Additive and Cooperative Effects on PC12 Cell Behavior. RSC Adv. 2016, 6, 97873-97881.

(32) Snyder, P. J.; Reddy, P.; Kirste, R.; LaJeunesse, D. R.; Collazo, R.; Ivanisevic, A. Noninvasive Stimulation of Neurotypic Cells Using Persistent Photoconductivity of Gallium Nitride. ACS Omega 2018, 3, 615-621.

(33) Berg, N. G.; Nolan, M. W.; Paskova, T.; Ivanisevic, A. Surface Characterization of Gallium Nitride Modified with Peptides before and after Exposure to Ionizing Radiation in Solution. Langmuir 2014, 30, 15477-15485. 